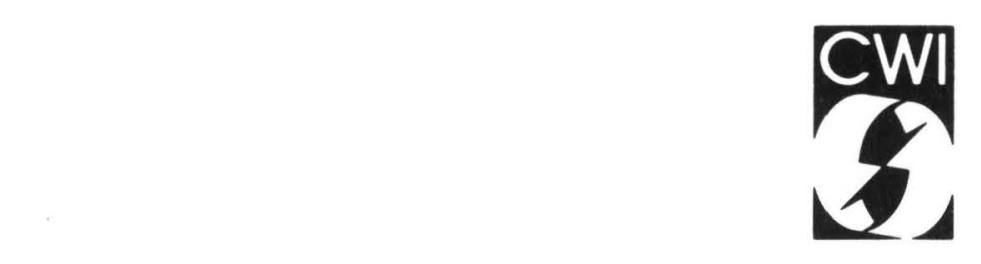

Centrum voor Wiskunde en Informatica Centre for Mathematics and Computer Science

J.F. Groote, F.W. Vaandrager

An efficient algorithm for branching bisimulation and stuttering equivalence 
The Centre for Mathematics and Computer Science is a research institute of the Stichting Mathematisch Centrum, which was founded on February 11, 1946 , as nonprofit institution aiming at the promotion of mathematics, computer science, and their applications. It is sponsored by the Dutch Government through the Netherlands Organization for the Advancement of Research (N.W.O.). 


\title{
An Efficient Algorithm for \\ Branching Bisimulation and Stuttering Equivalence
}

\author{
Jan Friso Groote \\ Frits Vaandrager \\ Centre for Mathematics and Computer Science \\ P.O. Box 4079, 1009 AB Amsterdam, The Netherlands \\ Email:jfg@cwi.nl\&tritsv@cwi.nl
}

\begin{abstract}
This paper presents an efficient algorithm for the Relational Coarsest Partition with Stuttering problem (RCPS). The RCPS problem is closely related to the problem of deciding stuttering equivalence on finite state Kripke structures (see Browne, ClaRke \& Grumberg [3]), and to the problem of deciding branching bisimulation equivalence on finite state labelled transition systems (see VAN GLABBEEK \& WEIJLAND [12]). If $n$ is the number of states and $m$ the number of transitions, then our algorithm has time complexity $O(n \cdot(n+m))$ and space complexity $O(n+m)$. The algorithm induces algorithms for branching bisimulation and stuttering equivalence which have the same complexity. Since for Kripke structures $m \leqslant n^{2}$, this confirms a conjecture of BroWNE, CLARKE \& GRUMBERG [3], that their $O\left(n^{5}\right)$-time algorithm for stuttering equivalence is not optimal.
\end{abstract}

Key Words and Phrases: labelled transition systems, branching bisimulation equivalence, Kripke structures, stuttering equivalence, divergence blind stuttering equivalence, relational coarsest partition problem (RCP), relational coarsest partition with stuttering problem (RCPS), decision algorithm.

1985 Mathematics Subject Classification: 68Q05,68Q20,68Q25, 68Q60.

1987 CR Categories: F.1.1, F.2.1, F.3.1.

Note: To appear in: Proceedings ICALP 90. The research of the authors was supported by RACE project no. 1046, Specification and Programming Environment for Communication Software (SPECS). The research of the second author was also supported by ESPRIT project no. 3006 CONCUR.

\section{INTRODUCTION}

In this paper we present an efficient algorithm for the Relational Coarsest Partition with Stuttering problem (RCPS). This problem is interesting because it is closely related to (1) deciding stuttering equivalence on finite Kripke structures, and (2) deciding branching bisimulation equivalence on finite labelled transition systems. Below we comment on these two problems separately.

1.1. Stuttering equivalence. Temporal logic model checking procedures have been successful in finding errors in relatively small network protocols and sequential circuits (for an overview see [5]). However, a serious problem for the model checking approach is the state explosion problem: in general, the number of states in the global state graph may grow exponentially with the number of components. In order to deal with this problem, it seems natural to hide details that do not need to be visible externally and merge those states that become indistinguishable. In the setting of temporal logic, hiding of 'details' is achieved by making it illegal to refer to these details in temporal logic formulas. If a program is constructed in a hierarchical fashion, then state explosion may be avoided by simplifying the components before computing the global state graph [6]. Our paper deals with the question how the idea of merging indistinguishable states can be implemented in the setting of the logic CTL*.

Report CS-R9001

Centre for Mathematics and Computer Science

P.O. Box 4079, 1009 AB Amsterdam, The Netherlands 
The computation tree logic $\mathrm{CTL}^{*}[10]$ is a very powerful temporal logic that combines both branching time and linear time operators. CTL [4] is a restricted subset of CTL ${ }^{*}$ that permits only branching time operators. One of the operators in $\mathrm{CTL}_{\mathrm{CTL}}{ }^{*}$, the nexttime operator $X$, has been subject to some criticism. LAMPORT [15] argues that in reasoning about concurrent systems, the nexttime operator may be dangerous since it refers to the global next state instead of the local next state. If, for this reason, one decides not to use the nexttime operator, then it becomes interesting to study the equivalences on states induced by the sets of formulas CTL $-X$ and $\mathrm{CTL}^{*}-X$. The idea is that two states that satisfy the same CTL $-X / \mathrm{CTL}^{*}-X$ formulas have the same relevant properties (and maybe some irrelevant ones as well) and are therefore identified. Browne, Clarke \& Grumberg [3] introduce the notion of stuttering equivalence on Kripke structures and prove that this equivalence characterizes both the equivalence induced by $\mathrm{CTL}-X$ and the equivalence induced by $\mathrm{CTL}^{*}-X$. They show that stuttering equivalence can be decided in polynomial time but give a rather high upper bound of $O\left(n^{5}\right)$ for the time complexity, where $n$ is the number of states of the Kripke structure. They conjecture the existence of a faster algorithm. The present paper confirms this conjecture: our algorithm for the RCPS problem solves stuttering equivalence in $O(m \cdot n)$ time, where $m$ is the number of transitions in the Kripke structure. Here, like in the rest of this paper, we assume $m \geqslant n$. The reader may drop this assumption if (s)he reads $m+n$ whenever we write $m$. In Kripke structures always $m \leqslant n^{2}$.

1.2. Branching bisimulation equivalence. VAN GLABBEEK \& WeiJland [12] introduce the notion of branching bisimulation equivalence on labelled transition systems. This equivalence resembles, but is finer than the observation equivalence of MiLner [17]. They argue that, unlike observation equivalence, branching bisimulation preserves the branching structure of processes, in the sense that it preserves computations together with the potentials in all intermediate states that are passed through, even if silent moves are involved. Besides this conceptual aspect, a number of other recent results indicate that branching bisimulation equivalence has very nice properties:

1. The corresponding congruence has a simple algebraic characterization [12]. In the setting of CCS, only a single axiom has to be added to the laws for strong bisimulation equivalence:

$$
a \cdot(\tau \cdot(x+y)+x)=a \cdot(x+y) .
$$

2. In contrast with observation equivalence, the resulting axiom system can be turned easily into a complete term rewriting system.

3. A modal characterization of branching bisimulation equivalence can be obtained by adding to Hennessy-Milner logic a kind of until operator [9].

4. Branching bisimulation equivalence can be characterized in terms of back and forth bisimulations [8]. This characterization induces a second modal characterization of branching bisimulation which is a variant of Hennessy-Milner logic with backward modalities [9].

5. Branching bisimulation equivalence is the natural analogue of stuttering equivalence in a setting where the transitions rather than the states are labelled. It is even possible to view $\mathrm{CTL}^{*}-X$ as a logic for branching bisimulation [9].

6. Unlike observation equivalence, branching bisimulation is preserved under refinement of actions in a setting without parallelism [13].

7. For a large class of processes, branching bisimulation and observation equivalence are the same [12]. We are not aware of any protocol that can be verified in the setting of observation equivalence but not in the stronger setting of branching bisimulation equivalence.

In this paper we show how our algorithm for RCPS can be easily transformed to an $O(m \cdot n)$ algorithm for deciding branching bisimulation equivalence $(O(m \log m+m \cdot n)$ if the set of labels is infinite or not fixed). 
1.3. Outline of paper. The structure of this paper is as follows. In Section 2 we present the RCPS problem and in Section 3 our algorithm to solve it. Section 4 describes how the algorithm can be used to decide stuttering equivalence and in Section 5 we show how a variant of the algorithm solves the problem of deciding branching bisimulation. Section 6 contains some concluding remarks. We conjecture that, at the price of a more complicated algorithm, the efficiency of our algorithm for RCPS can be slightly improved upon by incorporating ideas from the $O(m \log n)$ algorithm of PAIGE \& TARJAN [18] for the Relational Coarsest Partition problem (RCP). Also in Section 6, we compare the complexity of deciding branching bisimulation equivalence with the complexity of deciding observation equivalence.

\section{ACKNOWLEDGEMENTS}

We thank Robert de Simone and Didier Vergamini for helping us to obtain the test results of Section 6. We also thank Scott Smolka for proof-reading of an earlier version.

\section{THE RCPS PROBLEM}

Let $S$ be a set. A collection $\left\{B_{j} \mid j \in J\right\}$ of nonempty subsets of $S$ is called a partition of $S$ if $\cup_{j \in J} B_{j}=S$ and for $i \neq j: B_{i} \cap B_{j}=\varnothing$. The elements of a partition are called blocks. If $P$ and $P^{\prime}$ are partitions of $S$ then $P^{\prime}$ refines $P$, and $P$ is coarser than $P^{\prime}$, if any block of $P^{\prime}$ is included in a block of $P$. The equivalence $\sim_{P}$ on $S$ induced by a partition $P$ is defined by: $r \sim_{P} s \Leftrightarrow \exists B \in P: r \in B \wedge s \in B$.

The Relational Coarsest Partition with Stuttering problem (RCPS) can now be specified as follows:

Given: a nonempty, finite set $S$ of states, a relation $\rightarrow \subseteq S \times S$ of transitions, and an initial partition $P_{0}$ of $S$.

Find: the coarsest partition $P_{f}$ satisfying:

(i) $P_{f}$ refines $P_{0}$;

(ii) if $r \sim_{P_{f}} s$ and $r \rightarrow r^{\prime}$, then there is an $n \geqslant 0$ and there are $s_{0}, \ldots, s_{n} \in S$ such that:

- $s_{0}=s$;

- $\quad$ for all $0 \leqslant i<n: r \sim_{P_{f}} s_{i}$ and $s_{i} \rightarrow s_{i+1}$;

- $r^{\prime} \sim_{P_{f}} s_{n}$.

Below we show that a coarsest partition satisfying (i) and (ii) always exists; if it exists, then clearly it is unique.

\section{The Algorithm}

Next we will describe our algorithm for the RCPS problem. We fix a nonempty, finite set $S$ of states, a transition relation $\rightarrow$ and an initial partition $P_{0}$. Let $|S|=n$ and $|\rightarrow|=m$. For $B, B^{\prime} \subseteq S$ we define the set $\operatorname{pos}\left(B, B^{\prime}\right)$ as the set of states in $B$ from which, after some initial stuttering, a state in $B^{\prime}$ can be reached:

$$
\operatorname{pos}\left(B, B^{\prime}\right)=\left\{s \in B \mid \exists n \geqslant 0 \exists s_{0}, \ldots, s_{n}: s_{0}=s,\left[\forall i<n: s_{i} \in B \wedge s_{i} \rightarrow s_{i+1}\right] \text { and } s_{n} \in B^{\prime}\right\} .
$$

Call $B^{\prime}$ a splitter of $B$ and $\left(B, B^{\prime}\right)$ a splitting pair iff $\varnothing \neq \operatorname{pos}\left(B, B^{\prime}\right) \neq B$. Since $\operatorname{pos}(B, B)=B$, a block can never be a splitter of itself. If $P$ is a partition of $S$ and $B^{\prime}$ a splitter of $B$, define $\operatorname{Ref}_{P}\left(B, B^{\prime}\right)$ as the partition obtained from $P$ by replacing $B$ by $\operatorname{pos}\left(B, B^{\prime}\right)$ and $B-\operatorname{pos}\left(B, B^{\prime}\right)$. $P$ is stable with respect to a block $B^{\prime}$ if for no block $B, B^{\prime}$ is a splitter of $B . P$ is stable if it is stable with respect to all its blocks. Thus the RCPS problem consists of finding the coarsest stable partition that refines $P_{0}$.

Our algorithm maintains a partition $P$ that is initially $P_{0}$. The following refinement step is repeated as long as $P$ is not stable:

find $B, B^{\prime} \in P$ such that $B^{\prime}$ is a splitter of $B$;

$$
P:=\operatorname{Ref}_{P}\left(B, B^{\prime}\right)
$$


3.1. ThEOREM. The above algorithm terminates after at most $n-\left|P_{0}\right|$ refinement steps. The resulting partition $P_{f}$ is the coarsest stable partition refining $P_{0}$.

Proof. In order to see that the algorithm terminates, observe that after each iteration of the refinement step the number of blocks of $P$ has increased by one. Since a partition of $S$ can have at most $n$ blocks, termination will occur after at most $n-\left|P_{0}\right|$ iterations.

Next we show that the algorithm solves the RCPS problem. By induction on the number of refinement steps we prove that any stable refinement of $P_{0}$ is also a refinement of the current partition. Clearly the statement holds initially. Suppose it is true before a refinement step that refines a partition $P$ to a partition $Q$, using a splitting pair $\left(B, B^{\prime}\right)$. Let $R$ be any stable refinement of $P_{0}$ and let $C$ be a block of $R$. It is enough to show that $C$ is included in a block from $Q$. By induction hypothesis, we can assume that $C$ is included in a block $D$ of $P$. If $D \neq B$, then $D$ is a block of $Q$ and we are done. So suppose $D=B$. We have to show that either $C \subseteq \operatorname{pos}\left(B, B^{\prime}\right)$ or $C \subseteq B-\operatorname{pos}\left(B, B^{\prime}\right)$. Suppose that there are $r, s \in C$ with $r \in \operatorname{pos}\left(B, B^{\prime}\right)$ and $s \notin p o s\left(B, B^{\prime}\right)$. We derive a contradiction. There are $r_{0}, \ldots, r_{n}$ such that $r=r_{0}$, for all $i<n: r_{i} \in B \wedge r_{i} \rightarrow r_{i+1}$ and $r_{n} \in B^{\prime}$. Let $C_{0}, \ldots, C_{n}$ be the blocks of $R$ such that $r_{i} \in C_{i}$. Then $C_{0}=C$ and, by induction, for all $i<n: C_{i} \subseteq B$ and $C_{n} \subseteq B^{\prime}$. Now use the fact that $R$ is stable to construct a sequence $s_{0}, \ldots, s_{m}$ with $s_{0}=s$, for $i<m: s_{i} \in B \wedge s_{i} \rightarrow s_{i+1}$ and $s_{m} \in B^{\prime}$. This contradicts $s \notin \operatorname{pos}\left(B, B^{\prime}\right)$. Thus we have proved the induction step.

Below we describe an implementation of our algorithm. We show how to compute in $O(m)$ time whether or not a partition is stable. The computation is organized in such a way that if the partition is not stable, a counterexample, i.e. a splitting pair $\left(B, B^{\prime}\right)$, is produced. Next we show how to compute $\operatorname{Ref}_{P}\left(B, B^{\prime}\right)$ in $O(m)$ time. Since the number of iterations of the main loop is $O(n)$, this establishes a complexity of $O(m \cdot n)$ for the RCPS problem.

Efficient implementation of the algorithm requires some preprocessing. Let $P$ be a partition. We call a transition $s \rightarrow s^{\prime}$ inert with respect to $P$, or $P$-inert, iff $s \sim_{P} s^{\prime}$. If in the initial partition a set of states is strongly connected via inert transitions, then these states will be in the same block of the final partition: by definition of inert they are in the same block of the initial partition, and no refinement step will place two states from the set in a different block. As a preprocessing step in our algorithm we look for strongly connected components with respect to inert transitions in the initial partition and 'collapse' these components to one state. Here we can use the well-known $O(m)$ algorithm for finding strongly connected components in a directed graph (see for instance Ано, НорCROFT \& UlLman [1]). Thus it is sufficient to solve the RCPS problem in the case where $P_{0}$ contains no cycles of inert transitions.

For $B \subseteq S$, define the set bottom $(B)$ of bottom states of $B$ by:

$$
\operatorname{bottom}(B)=\{r \in B \mid \forall s: r \rightarrow s \Rightarrow s \notin B\} .
$$

If $P$ is a partition, then the set bottom $(P)$ of bottom states of $P$ is given by:

$$
\operatorname{bottom}(P)=\bigcup_{B \in P} \operatorname{bottom}(B) \text {. }
$$

The following two observations play a crucial role in the implementation of our algorithm:

3.2. Lemma. Let $P$ be a refinement of $P_{0}$ and let $B, B^{\prime} \in P$. Then $B^{\prime}$ is a splitter of $B$ iff

(1) $B \neq B^{\prime}$,

(2) for some $r \in B$ and $r^{\prime} \in B^{\prime}: r \rightarrow r^{\prime}$, and

(3) there is an $s \in \operatorname{bottom}(B)$ such that for no $s^{\prime} \in B^{\prime}: s \rightarrow s^{\prime}$,

Proof. " $\Rightarrow$ " Suppose $B^{\prime}$ is a splitter of $B$. Then $B \neq B^{\prime}$ because a block can never split itself. By definition of a splitter: $\varnothing \neq \operatorname{pos}\left(B, B^{\prime}\right)$. Thus $r \rightarrow r^{\prime}$ for some $r \in B$ and $r^{\prime} \in B^{\prime}$. Suppose that for every 
bottom state $s$ of $B$ there is an $s^{\prime} \in B^{\prime}$ with $s \rightarrow s^{\prime}$. We derive a contradiction. Pick an element $t \in B$. Since $P_{0}$ contains no cycle of inert transitions, $P$ does not contain such a cycle either. Thus there must be a path of inert transitions from $t$ to a bottom state $t^{\prime}$ of $B$. Since for some $t^{\prime \prime} \in B^{\prime}$ we have $t^{\prime} \rightarrow t^{\prime \prime}, t$ is in $\operatorname{pos}\left(B, B^{\prime}\right)$. But since $t$ was chosen arbitrarily, this means that $\operatorname{pos}\left(B, B^{\prime}\right)=B$. This contradicts the fact that $B^{\prime}$ is a splitter of $B$.

" $\Leftarrow$ " Suppose that $B$ and $B^{\prime}$ satisfy condition (1), (2) and (3). Then $B^{\prime}$ is a splitter of $B$ : $\operatorname{pos}\left(B, B^{\prime}\right) \neq \varnothing$ because of (2), and $\operatorname{pos}\left(B, B^{\prime}\right) \neq B$ because of (1) and (3).

3.3. Lemma. Let $P, R$ be partitions such that $R$ refines $P$, and $P$ and $R$ have the same bottom states. Let $B$ be a block of both $P$ and $R$ such that $P$ is stable with respect to $B$. Then $R$ is stable with respect to $B$.

Proof. Let $P, R$ and $B$ be as above. Pick a block $B^{\prime}$ of $R$. Suppose that $B$ is a splitter for $B^{\prime}$. We will derive a contradiction. Application of Lemma 3.2 gives: (1) $B^{\prime} \neq B$, (2) for some $r \in B^{\prime}$ and $r^{\prime} \in B$ : $r \rightarrow r^{\prime}$, and (3) there is an $s \in$ bottom $\left(B^{\prime}\right)$ such that for no $s^{\prime} \in B: s \rightarrow s^{\prime}$. Now use that $B^{\prime}$ is included in some block $C$ of $P$. Clearly $C \neq B$. Moreover we can find $r \in C$ and $r^{\prime} \in B$ with $r \rightarrow r^{\prime}$. Since $\operatorname{bottom}(P)=\operatorname{bottom}(R)$ we have $\operatorname{bottom}\left(B^{\prime}\right) \subset \operatorname{bottom}(C)$. Thus we can find an $s \in$ bottom $(C)$ such that for no $s^{\prime} \in B: s \rightarrow s^{\prime}$. Now apply Lemma 3.2 to conclude that $B$ is a splitter for $C$, which is a contradiction.

3.4. Remark. In the setting of the Relational Coarsest Partition problem, stability is inherited under refinement in general; that is, if $R$ is a refinement of $P$ and $P$ is stable with respect to $B$, then so is $R$. The $O(m \log n)$ algorithm of PAIGE \& TARJAN [18] depends crucially on this property.

In the case of the RCPS problem stability is in general not inherited under refinement; the condition in Lemma 3.3 that $P$ and $R$ have the same bottom states cannot be dropped. An example is presented in Figure 1 below. If $P=\{\{s, t, u\},\{v, w\}\}$ and $R=\{\{s, t\},\{u\},\{v, w\}\}$, then $P$ is stable wrt $\{v, w\}$ but $R$ is not. As a consequence the idea behind the PAIGE \& TARJAN [18] algorithm cannot be applied directly to solve the RCPS problem.

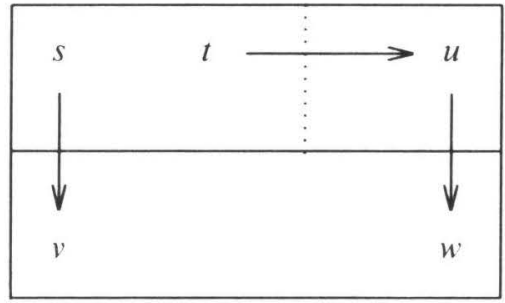

FIGURE 1.

3.5. The implementation. In the implementation of the algorithm, there is for each block, state and transition a corresponding record of type block, state resp. transition (see Figure 2). We identify a block, state and transition with the record representing it. There are two doubly linked lists, tobeprocessed and stable, of blocks. A block $B$ is in stable when the current partition is stable with respect to $B$. Otherwise $B$ is in the list tobeprocessed. Initially, all blocks of $P_{0}$ are in the list tobeprocessed and the list stable is empty. Each block $B$ points to a list of bottom states in $B$ and to a list of the nonbottom states in $B$. We assume that whenever $s \rightarrow s^{\prime}$ for $s, s^{\prime} \in B-\operatorname{bottom}(B), s$ is after $s^{\prime}$ in the list of non-bottom states. Initially, the division of states in bottom states and non-bottom states, and also the ordering on the non-bottom states, can be accomplished by a standard depth first search algorithm using $O(m)$ time and space (see for instance AHO, HopCrOFT \& Ullman [1]). Each state contains a pointer to the block of which it is an element. Each transition contains two pointers to resp. its starting state and its target state. Each non-bottom state points to a list of the inert transitions 


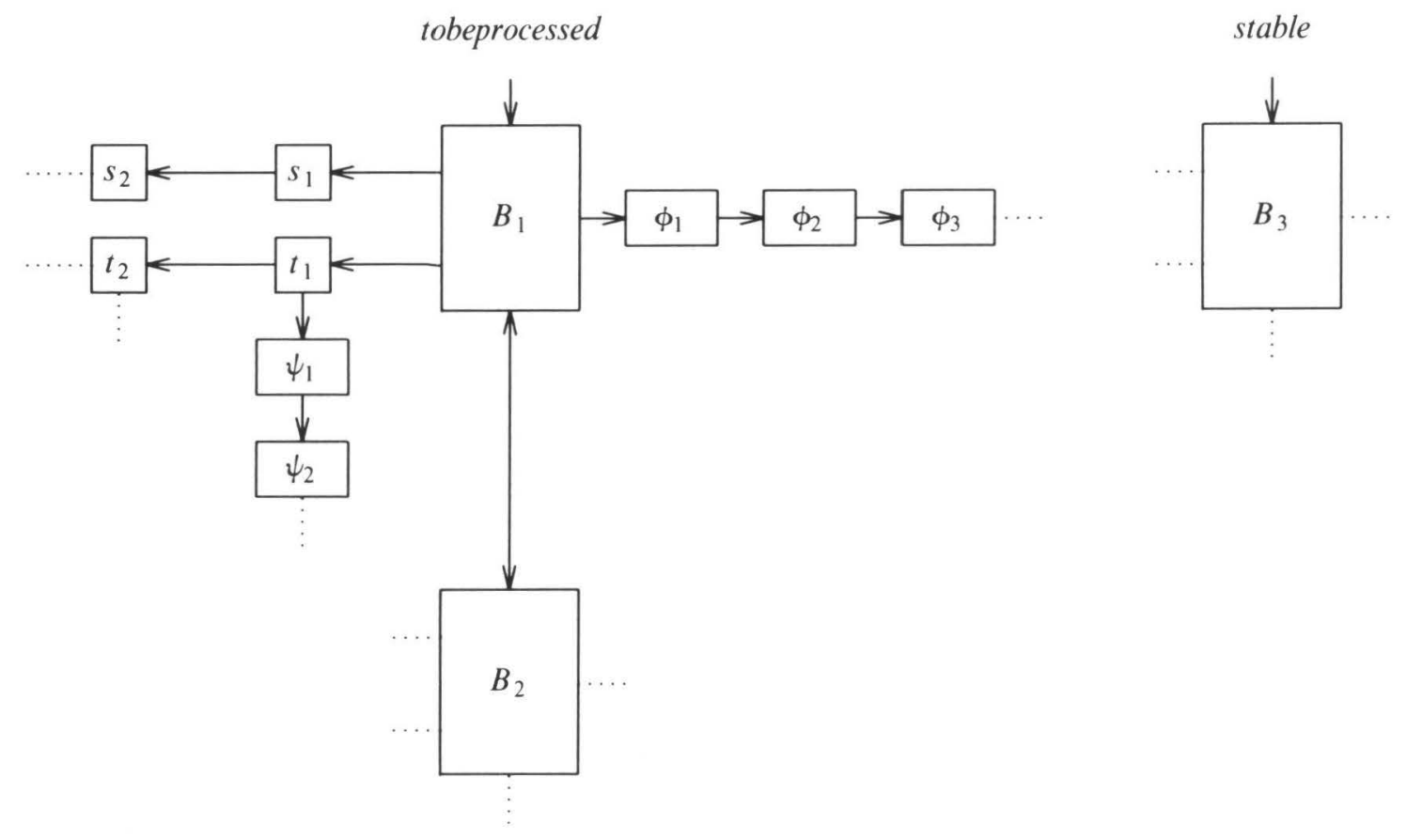

- $B_{1}, B_{2}, B_{3}, \ldots$ are the blocks of the current partition.

- $s_{1}, s_{2}, \ldots$ are the bottom states in block $B_{1}$.

- $\quad t_{1}, t_{2}, \ldots$ are the non-bottom states in block $B_{1}$.

- $\phi_{1}, \phi_{2}, \phi_{3}, \ldots$ are the non-inert transitions that end in block $B_{1}$.

- $\quad \psi_{1}, \psi_{2}, \ldots$ are the inert transitions that start in state $t_{1}$.

FIGURE 2.

starting in this state. Each block points to a list of the non-inert transitions that end in this block. Each state and each block has an auxiliary field flag of type boolean, which is 0 initially. Moreover we use an auxiliary variable $B L$ of type list of pointers to a block, which is empty initially.

Next we describe how to find out in $O(m)$ time whether a partition is stable. Let $B^{\prime}$ be a block in tobeprocessed. Scan the list of non-inert transitions which end in $B^{\prime}$. When some transition is visited, the flag of the starting state is raised (i.e. the value 1 is assigned to the field flag of the starting state). If the flag of the block to which the starting state belongs has not yet been raised, then we do so and append a pointer to this block to the list $B L$. After having scanned all non-inert transitions ending in $B^{\prime}$, we consider the list $B L$. This list contains references to all blocks, different from $B^{\prime}$, which contain a state from which a state from $B^{\prime}$ can be reached. There is at most one reference to each block in the list. Remove the first element from the list $B L$ and consider the block $B$ to which this element refers. By Lemma 3.2, $B^{\prime}$ is a splitter of $B$ iff there is a bottom state $s$ of $B$ such that for no $s^{\prime} \in B^{\prime}: s \rightarrow s^{\prime}$. So in order to find out whether $B^{\prime}$ is a splitter of $B$ we only have to check whether the flag of all bottom states in $B$ is raised.

Suppose that $B^{\prime}$ is a splitter of $B$. In this case we remove $B$ from the linked list of blocks in which it occurs (in general this can be either the list tobeprocessed or the list stable), and insert two new blocks $B_{1}$ and $B_{2}$ in the list tobeprocessed. The flag fields of the new blocks are set to 0 . All bottom states of $B$ with a raised flag become bottom states of $B_{1}$, the other bottom states of $B$ become bottom states of $B_{2}$. Next we scan the non-bottom states of $B$. If for some non-bottom state the flag is not raised 
and if none of the outgoing $P$-inert transitions leads to a state in $B_{1}$, then this state becomes a nonbottom state of $B_{2}$ (here we use the ordering on the list of non-inert states in the old partition). In this case the outgoing inert transitions of this state remain the same. Otherwise, the state is placed in $B_{1}$. It may be that certain transitions which were inert in the old partition lead to a state in $B_{2}$. In that case they have to be moved to the list of non-inert transitions which end in $B_{2}$. It may be that a state which is not a bottom state in the old partition becomes a bottom state in the new partition, just because no inert transitions are left. If, in a refinement, a non-bottom state becomes a bottom state, then (cf. Lemma 3.3) we append the list stable to the list iobeprocessed and make stable empty. The non-inert transitions ending in $B$ are distributed in the obvious way over $B_{1}$ and $B_{2}$.

If $B^{\prime}$ is not a splitter of $B$, or if it is a splitter and we have carried out the splitting as described above, then we consider the block referred to by the new first element of list $B L$ and check whether $B^{\prime}$ is splitter for that block, etc. When we have dealt with all element of list $B L$ then we know that for no block in the current partition $B^{\prime}$ is a splitter. We move $B^{\prime}$ from the list tobeprocessed to the list stable, reinitialize all flags by an additional scan of the non-inert transitions with an end state in $B^{\prime}$, and we apply the same procedure for a next block in tobeprocessed, etc. If tobeprocessed is empty then we know that the current partition is stable.

One can easily check that in $O(m)$ time we either have found a splitter and refined the partition, or we have established that the current partition is stable. Moreover the space complexity is $O(m)$. Thus we have the following theorem:

\subsection{THEOREM. The RCPS problem can be decided in $O(m \cdot n)$ time, using $O(m)$ space.}

3.7. Remark. The implementation may be simplified slightly by eliminating the stable list. However, since the stable list provides a very simple way to avoid a lot of work (in our trial implementation the time performance increased with more than a factor 2), we decided to include it in the above description.

\section{Stuttering equivalence}

In this section we show how a solution of the the RCPS problem can be used to decide stuttering equivalence on finite Kripke structures. Let AP be a set of atomic proposition names.

4.1. Definition. A Kripke structure is a triple $\mathcal{H}=(S, \rightarrow, \varrho)$ where $S$ is a set of states, $\rightarrow \subseteq S \times S$ is the transition relation and $\mathrm{L}: S \rightarrow 2^{\mathrm{AP}}$ is the proposition labelling. A Kripke structure is finite if the set of states is finite and for each state the set of associated proposition names is finite.

4.2. Definition. Let $\mathcal{H}=(S, \rightarrow, \mathcal{L})$ be a Kripke structure. A relation $R \subseteq S \times S$ is called a divergence blind stuttering bisimulation if it is symmetric and whenever $r R s$ then:

(i) $\mathcal{L}(r)=\mathcal{Q}(s)$ and

(ii) if $r \rightarrow r^{\prime}$ then there exist $s_{0}, \ldots, s_{n} \in S(n \geqslant 0)$ such that $s=s_{0}$, for all $0 \leqslant i<n: s_{i} \rightarrow s_{i+1} \wedge r R s_{i}$, and $r^{\prime} R s_{n}$.

Two states $r, s \in S$ are divergence blind stuttering equivalent, notation $h: r \leftrightarrow_{d b s} s$ or just $r \hookrightarrow_{d b s} s$, iff there exists a divergence blind stuttering bisimulation relation relating $r$ and $s$. One can easily check that divergence blind stuttering equivalence is indeed an equivalence relation.

Let $\xi_{i}=(S, \rightarrow, E)$ be a finite Kripke structure with $|S|=n$ and $|\rightarrow|=m$. In order to determine whether two states in $S$ are divergence blind stuttering equivalent, one can use our RCPS algorithm as follows.

The initial partition $P_{0}$ is constructed by putting all states with the same labels in the same block. Assuming that the set AP is finite and fixed, the initial partition can be computed in $O(n)$ time using 
a lexicographic sorting method [1]. If lexicographic sorting is not feasible, it can be computed in $O(n \log n)$ time. Next the RCPS algorithm is used to compute the coarsest stable partition $P_{f}$ that refines $P_{0}$. This takes $O(m \cdot n)$ time. The following theorem says that partition $P_{f}$ solves our problem.

4.3. TheOREM. Two states are in the same block of $P_{f}$ exactly when they are divergence blind stuttering equivalent.

Proof. Suppose $r, s \in B$ for some block $B$ in $P_{f}$. Let $R_{f}$ be the relation that relates two states iff they are in the same block of $P_{f}$. Clearly $r R_{f} s$. We show that $R_{f}$ is a divergence blind stuttering bisimulation. Let $p, q \in S$ with $p R_{f} q$. As $P_{f}$ refines $P_{0}, \mathscr{L}(p)=\mathscr{E}(q)$. Moreover, condition (ii) of Definition 4.2 holds as it exactly coincides with the condition (ii) in the RCPS problem. Hence $R_{f}$ is a divergence blind stuttering bisimulation and $r$ and $s$ are divergence blind stuttering equivalent.

Let $P_{s}$ be the partition of $S$ induced by $\Theta_{d b s}$. By definition of divergence blind stuttering $P_{s}$ refines $P_{0}$. Moreover, $P_{s}$ is stable. As $P_{f}$ is the coarsest stable partition refining $P_{0}, P_{s}$ refines $P_{f}$.

Thus the time complexity of deciding divergence blind stuttering equivalence is at most $O(m \cdot n+n \log n)=O(m \cdot n)($ remember $m \geqslant n)$.

In De Nicola \& VaAndrager [9] it is shown that for finite Kripke structures divergence blind stuttering equivalence coincides with the equivalence induced by $\mathrm{CTL}-X / \mathrm{CTL}^{*}-X$ formulas if one quantifies over all paths in the Kripke structure (the finite as well as the infinite ones). If one only quantifies over the infinite paths, then this leads to the following stuttering equivalence:

4.4. Definition. Let $\mathscr{H}=(S, \rightarrow$, 2$)$ be a Kripke structure. Let $s_{0}$ be a state not in $S$ and let $p_{0}$ be an atomic proposition such that for all $s$ in $S: p_{0} \notin Q(s)$. Define a Kripke structure $\mathcal{R}^{\prime \prime}=\left(S^{\prime}, \rightarrow^{\prime}, L^{\prime \prime}\right)$ by:

- $S^{\prime}=S \cup\left\{s_{0}\right\}$,

- $\quad \rightarrow^{\prime}=\longrightarrow \cup\left\{\left(s, s_{0}\right) \mid s \in S\right.$ has no outgoing transition or occurs on a cycle of states which all have

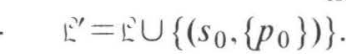
the same label\},

Two states $r, s \in S$ are stuttering equivalent if in $h^{\prime}: r \Theta_{d b s} s$ (Note that this definition does not depend on the particular choice of state $s_{0}$ and atomic proposition $p_{0}$ ).

For finite Kripke structures the stuttering equivalence as defined above coincides with the equivalence induced by CTL $-X$ and $\mathrm{CTL}^{*}-X$ if one quantifies over infinite paths [9]. Since Browne, Clarke \& GRUMBerg [3] proved the same result for their version of stuttering equivalence, both notions agree. For finite Kripke structures the transformation of Definition 4.4 can be accomplished in $O(m)$ time. Thus our algorithm for RCPS can be used to decide stuttering equivalence in $O(m \cdot n)$ time.

5. BRANCHING BISIMULATION EQUIVALENCE

The RCPS algorithm cannot be used directly for deciding branching bisimulation equivalence. We have to generalize RCPS to the case where transitions have labels.

5.1. The Generalized Relational Coarsest Partition with Stuttering problem (GRCPS) is given by:

Given: A nonempty, finite set $S$ of states, a finite set $A$ of labels containing the silent step $\tau$, a relation $\rightarrow \subseteq S \times A \times S$ of transitions and an initial partition $P_{0}$ of $S$.

Find: the coarsest partition $P_{f}$ satisfying:

(i) $P_{f}$ refines $P_{0}$.

(ii) if $r \sim_{P_{f}} s$ and $r \stackrel{a}{\rightarrow} r^{\prime}$, then either $a=\tau$ and $r^{\prime} \sim_{P_{f}} s$, or there is an $n \geqslant 0$ and there are $s_{0}, \ldots, s_{n}, s^{\prime}$ such that $s_{0}=s$, for all $0<i \leqslant n:\left[r \sim_{P_{f}} s_{i} \wedge s_{i-1} \stackrel{\tau}{\longrightarrow} s_{i}\right], s_{n} \stackrel{a}{\longrightarrow} s^{\prime}$ and $r^{\prime} \sim_{P_{f}} s^{\prime}$. 
5.2. We now present our algorithm for the GRCPS-problem. This algorithm is a minor modification of the algorithm for the RCPS-problem. Therefore, we will not describe it in detail but only sketch the differences. We fix $S, A, \rightarrow$ and $P_{0}$ and, as usual, write $|S|=n$ and $|\rightarrow|=m$.

For $B, B^{\prime} \subseteq S$ and $a \in A$, the set $\operatorname{pos}_{a}\left(B, B^{\prime}\right)$ is defined by:

$$
\operatorname{pos}_{a}\left(B, B^{\prime}\right)=\left\{s \in B \mid \exists n \geqslant 0 \exists s_{0}, \ldots, s_{n} \in B \quad \exists s^{\prime} \in B^{\prime}: s_{0}=s,\left[\forall 0<i \leqslant n: s_{i-1} \stackrel{\tau}{\longrightarrow} s_{i}\right] \text { and } s_{n} \stackrel{a}{\longrightarrow} s^{\prime}\right\} .
$$

We say that $B^{\prime}$ is a splitter of $B$ with respect to $a$ iff $B \neq B^{\prime}$ or $a \neq \tau$, and $\varnothing \neq p_{a}\left(B, B^{\prime}\right) \neq B$. If $P$ is a partition of $S$ and $B^{\prime}$ is a splitter of $B$ with respect to $a$, then $\operatorname{Ref}_{P}^{a}\left(B, B^{\prime}\right)$ is the partition $P$ where $B$ is replaced by $\operatorname{pos}_{a}\left(B, B^{\prime}\right)$ and $B-\operatorname{pos}_{a}\left(B, B^{\prime}\right)$. $P$ is stable with respect to a block $B^{\prime}$ if for no block $B$ and for no action $a, B^{\prime}$ is a splitter of $B$ wrt $a . P$ is stable if it is stable with respect to all its blocks.

The algorithm maintains a partition $P$ that is initially $P_{0}$. It repeats the following step, until $P$ is stable:

find blocks $B, B^{\prime} \in P$ and a label $a \in A$ such that $B^{\prime}$ is a splitter of $B$ with respect to $a$; $P:=\operatorname{Ref}_{P}^{u}\left(B, B^{\prime}\right)$.

5.2.1. THEOREM. The above algorithm for the GRCPS problem terminates after at most $n-\left|P_{0}\right|$ refinement steps. The resulting partition $P_{f}$ is the coarsest stable partition refining $P_{0}$.

Proof. Similar to the proof of Theorem 3.1.

We must now show that one can find a splitter $B^{\prime}$ with respect to some label $a$ in time $O(m)$ or find in $O(m)$ time that no such splitter exists. Moreover, a refinement must be carried out in $O(m)$ time. To this purpose we use the data structure of the RCPS algorithm. But now a transition $s \stackrel{a}{\longrightarrow} s^{\prime}$ is called ( $P$-)inert if $s \sim_{P} s^{\prime}$ and $a=\tau$, and a state $s \in B$ is a bottom state of $B$ if $s \in B$ and there is no $s^{\prime} \in B$ such that $s \stackrel{\tau}{\longrightarrow} s^{\prime}$. The data structure is initialized in the same way as for the RCPS algorithm. However, the non-inert transitions ending in a block $B$ are grouped on label, i.e. all transitions with the same label are in subsequent records in the list. If there are non-inert transitions with a label $\tau$ ending in a block $B$, then they are at the beginning of the list. This facilitates adding inert transitions that become non-inert after a refinement at the beginning of the transition list. Grouping of the transitions has time complexity $O(m \log m)$ (heapsort) or $O(|A|+m)$ (bucket sort).

The following lemmas are the counterparts of Lemma 3.1 and 3.2. As the proofs are similar, they are omitted.

5.2.2. Lemma. Let $P$ be a refinement of $P_{0}$ and let $B, B^{\prime} \in P$ and $a \in A$. Then $B^{\prime}$ is a splitter of $B$ with respect to a iff

1) $a \neq \tau$ or $B \neq B^{\prime}$,

2) for some $r \in B$ and $r^{\prime} \in B^{\prime}: r \stackrel{a}{\longrightarrow} r^{\prime}$, and

3) there is a bottom state $s$ of $B$ such that for no $s^{\prime} \in B^{\prime}: s \stackrel{a}{\longrightarrow} s^{\prime}$.

5.2.3. Lemma. Let $P, R$ be partitions such that $R$ refines $P$, and $P$ and $R$ have the same bottom states. Let $B$ be a block of both $P$ and $R$ such that $P$ is stable with respect to $B$. Then $R$ is stable with respect to $B$.

A splitter can be found in the same way as in the RCPS algorithm. Continue the following step until the list tobeprocessed is empty or a splitter has been found. Consider a block $B$ from the list tobeprocessed. Consider subsequently all groups $\Phi$ of non-inert transitions ending in $B$ with the same label $a$, set the flag field of the starting states of transitions in $\Phi$ and construct $B L$. A copy of $\Phi$ is maintained for resetting the flags. Then check stability of all blocks $B^{\prime}$ in $B L$ with respect to $B$ and label $a$ and split $B^{\prime}$ if necessary. Due to Lemma 5.2.2 and Lemma 5.2.3 this can be performed in exactly the same 
way as in the RCPS case. Reset the flags of the states using the copy of $\Phi$. If $B$ splits itself into blocks $B_{1}$ and $B_{2}$, it is not necessary to check more transitions ending in $B$, as they must again be checked for $B_{1}$ and $B_{2}$. If all incoming transitions in block $B$ have been checked, if $B$ is not split and if there is no new bottom state, move $B$ from tobeprocessed to stable.

5.3. Branching bisimulation is mostly defined on labelled transition systems (LTS's). The GRCPSalgorithm can be used to decide branching bisimulation on finite LTS's.

5.3.1. Definition. A labelled transition system $(L T S)$ is a triple $P=(S, A, \rightarrow)$ with $S$ a set of states, $A$ a set of labels containing the silent step $\tau$, and $\rightarrow \subseteq S \times A \times S$ a transition relation. 2 is called finite if both $S$ and $A$ are finite.

5.3.2. Definition [12]. Let $\mathscr{Q}=(S, A, \rightarrow)$ be a LTS. Let $\Rightarrow$ be the transitive and reflexive closure of $\stackrel{\tau}{\rightarrow}$. A relation $R \subseteq S \times S$ is a branching bisimulation if it is symmetric and whenever $r R s$ and $r \stackrel{a}{\rightarrow} r^{\prime}$, then either $a=\tau$ and $r^{\prime} R s$, or there exist $s_{1}, s^{\prime}$ such that $s \Rightarrow s_{1} \stackrel{a}{\rightarrow} s^{\prime}$ and $r R s_{1}$ and $r^{\prime} R s^{\prime}$.

Two states $r, s \in S$ are branching bisimilar, notation $r \leftrightarrow_{b} s$, if there exists a branching bisimulation relation relating $r$ and $s$.

We could have strengthened this definition by requiring all intermediate states in $s \Rightarrow s_{1}$ to be related with $r$. The following lemma implies that this would lead to the same equivalence relation.

5.3.3. Lemma (cf. Lemma 1.3 of [12]). Let $Q=(S, A, \rightarrow)$ be a LTS and let for some $n>0$, $r_{0} \stackrel{\tau}{\rightarrow} r_{1} \stackrel{\tau}{\rightarrow} . . \stackrel{\tau}{\rightarrow} r_{n-1} \stackrel{\tau}{\rightarrow} r_{n}$ be a path in Eith $r_{0} \leftrightarrow_{b} r_{n}$. Then for all $0 \leqslant i \leqslant n: r_{0} \leftrightarrow_{b} r_{i}$.

5.3.4. TheOREM. Let $E=(S, A, \rightarrow)$ be a finite LTS. Let $P_{f}$ be the final partition obtained after applying the GRCPS algorithm on an initial partition containing only block $S$. Then $\sim_{P_{f}}=\leftrightarrows_{b}$.

Proof. " $\subseteq$ " Using Theorem 5.2.1 it follows that $\sim_{P_{f}}$ is a branching bisimulation relation.

$" \supseteq " \leftrightarrow_{b}$ induces a stable partition on $S$ (use Lemma 5.3.3). As $P_{f}$ is the coarsest stable partition, $\sim_{P_{f}} \supseteq \biguplus_{b}$.

So in order to compute whether two states in a finite LTS are branching bisimilar we can apply the GRCPS algorithm with as initial partition the partition containing the set of states as only block. This takes $O(m \log m+m \cdot n)$ resp. $O(|A|+m \cdot n)$ time.

\section{Concluding Remarks}

6.1. Lower bounds. So, is our $O(m \cdot n)$ algorithm for the RCPS problem optimal? We do not think so. In fact we conjecture that our algorithm can be slightly improved upon by incorporating ideas behind the $O(m \log n)$ algorithm of PAIGE \& TARJAN [18] for the RCP problem. Let $m_{i}$ be the number of inert transitions in the initial partition and let $n_{i}$ be the number of states which have an outgoing inert transition in the initial partition but are bottom states in the final partition. We have the following conjecture:

6.1.1. Conjecture. The RCPS problem can be decided in $O\left(m \cdot n_{i}+m_{i} \cdot n+m \log n\right)$ time, using $O(m)$ space.

As already observed in Remark 3.4, stability is not inherited under refinement in general: problems arise when, in a refinement, a non-bottom state becomes a bottom state. This situation can occur $n_{i}$ times. The summand $m \cdot n_{i}$ in the expression above corresponds to the additional amount of work that has to be done to deal with these situations. At present we do not see how to avoid scanning all inert 
transitions when we do a refinement step. This explains the summand $m_{i} \cdot n$. If there are no inert transitions, then the algorithm which we conjecture is as efficient as the PAIGE \& TARJAN [18] algorithm for the RCP problem. However, often $m_{i}$ will be of the same order as $m$. In that case the order of complexity equals the one of our $O(m \cdot n)$ algorithm. For this reason, and also because the algorithm which we conjecture is rather complex (it combines the techniques of PAIGE \& TARJAN [18] with the techniques of our $O(m \cdot n)$ algorithm), we decided to concentrate first on a clear exposition of the $O(m \cdot n)$ algorithm.

6.2. Branching bisimulation versus observation equivalence. In a sense, branching bisimulation equivalence can be viewed as an alternative to observation equivalence. Thus it is interesting to compare the complexities of deciding these equivalences. First consider the situation where the set $A$ of labels is fixed (so $O(m) \leqslant O\left(n^{2}\right)$ ). All known algorithms for deciding observation equivalence (see e.g. $[2,14])$ work in two phases. First a transitive closure algorithm is used to compute the so-called double arrow relation. With a simple algorithm (see e.g. [1]) this takes $O\left(n^{3}\right)$ time. The result of the transitive closure is a new LTS with at most $O\left(n^{2}\right)$ more edges than the original LTS. Next a variant of the PAIGE \& TARJAN [18] algorithm is used to decide strong bisimulation equivalence on the new LTS. This takes $O\left(n^{2} \log n\right)$ time. The resulting complexity in this case for deciding observation equivalence is $O\left(n^{3}\right)$, which is the same as the complexity of our algorithm. Now there are numerous sub-cubic transitive closure algorithms in the literature (see e.g. [7] for an $O\left(n^{2.376}\right)$ algorithm). These algorithms tend to be practical only for large values of $n$. Still we have that if the set of labels is fixed, the number of states is large and the number of transitions is of order $O\left(n^{2}\right)$, observation equivalence can be decided faster than branching bisimulation if one uses these sub-cubic algorithms.

However, things change if one does not fix the set of labels. Since one has to compute the double arrow relation for all labels that occur in the LTS, the complexity of computing the double arrow relation then becomes $O\left(m \cdot n^{2.376}\right)$ (at least, we do not know any faster solution). In that case our algorithm for branching bisimulation is more efficient.

6.3. A trial implementation. Clearly, the issue of comparing the complexities of observation equivalence and branching bisimulation is nontrivial and the analysis above does not give very much insight into the performance of our algorithm in practical applications. Therefore, we wrote a trial implementation in Pascal and compared the performance of this implementation with the performance of AUTO [19] and Aldébaran [11], as far as we know the two fastest tools currently available for deciding observation equivalence.

The process we used for our tests was the 'scheduler' as described by MiLner [16]. This scheduler schedules $k$ processes in succession modulo $k$, i.e. after process $k$ process 1 is reactivated again. However, a process must never be reactivated before it has terminated.
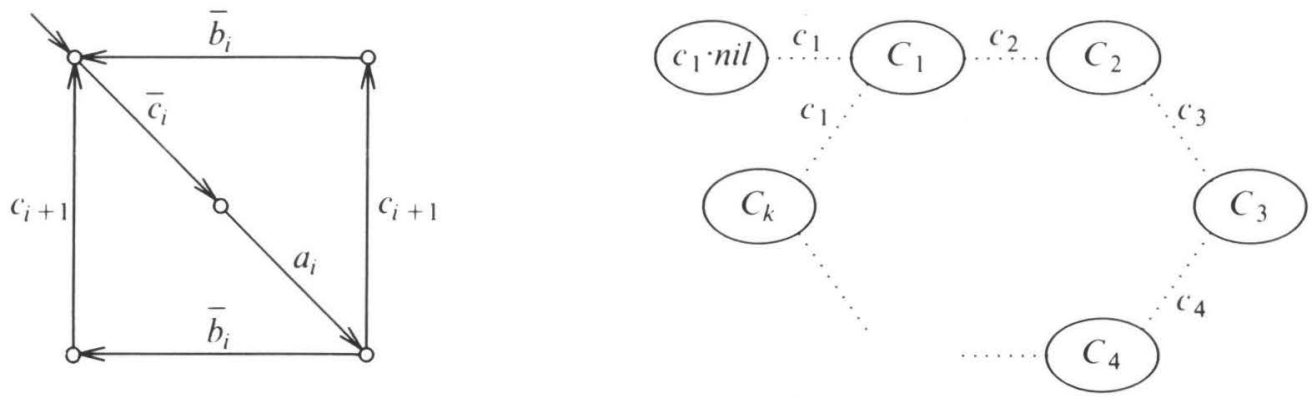

FIGURE 3.

The scheduler is constructed of $k$ cyclers $C_{1} \ldots, C_{k}$, where cycler $C_{i}$ takes care of process $i$. The left part of Figure 3 shows the transition system for cycler $C_{i}$. In the right part the architecture of the 
scheduler is depicted. The dotted lines indicate where the cyclers synchronize. Cycler $C_{i}$ first receives a signal $\bar{c}_{i}$ which indicates that it may start. It then activates process $i$ via an action $a_{i}$. Next, it waits for termination of process $i$. indicated by $\bar{b}_{i}$, and in parallel, it informs the next cycler that it may start. Finally, the cycler returns to its initial state. In a CCS-like language cycler $C_{i}$ is described by:

$$
C_{i}=\bar{c}_{i} \cdot a_{i} \cdot\left(\bar{b}_{i} \mid c_{i+1}\right) \cdot C_{i}
$$

The complete scheduler for $k$ processes is described by:

$$
S c h_{k}=\left(c_{1} \cdot \text { nil }\left|C_{1}\right| \cdots \mid C_{k}\right) \backslash c_{1} \cdots \backslash c_{k} .
$$

Here $c_{1} \cdot$ nil is an auxiliary process that starts the first cycler.

The results of experiments with schedulers of different size are given in Table 1 . Here $k$ is the number of cyclers per scheduler. The second and third column give the number of states resp. transitions of the corresponding transition system. Then we give the time necessary to calculate the bisimulation equivalence classes of the schedulers for Aldébaran, AUTO and our trial implementation (BB). We give these figures not only for the case where labels $a_{i}$ and $b_{i}$ are both visible, but also for the case where actions $b_{i}$ are hidden (i.e. renamed into $\tau$ ).

\begin{tabular}{|r|r|r||r|r|r|r||r|r|r|r||}
\hline \multicolumn{9}{|c||}{} & \multicolumn{3}{|||}{ both $a_{i}$ and $\bar{b}_{i}$ visible } & \multicolumn{3}{l|}{ only $a_{i}$ visible } \\
\hline$k$ & states & trans. & AUTO & Aldéb. & BB & eq.cl. & AUTO & Aldéb. & BB & eq.cl. \\
\hline 4 & 97 & 241 & $0.5 \mathrm{~s}$ & $0.26 \mathrm{~s}$ & $0.07 \mathrm{~s}$ & 64 & $0.4 \mathrm{~s}$ & $0.15 \mathrm{~s}$ & $0.02 \mathrm{~s}$ & 4 \\
5 & 241 & 721 & $1.9 \mathrm{~s}$ & $0.88 \mathrm{~s}$ & $0.3 \mathrm{~s}$ & 160 & $1.1 \mathrm{~s}$ & $0.6 \mathrm{~s}$ & $0.07 \mathrm{~s}$ & 5 \\
6 & 577 & 2017 & $8.0 \mathrm{~s}$ & $2.6 \mathrm{~s}$ & $0.9 \mathrm{~s}$ & 384 & $3.3 \mathrm{~s}$ & $1.9 \mathrm{~s}$ & $0.2 \mathrm{~s}$ & 6 \\
7 & 1345 & 5377 & $38 \mathrm{~s}$ & $7.2 \mathrm{~s}$ & $2.5 \mathrm{~s}$ & 896 & $12 \mathrm{~s}$ & $6.9 \mathrm{~s}$ & $0.5 \mathrm{~s}$ & 7 \\
8 & 3073 & 13825 & $201 \mathrm{~s}$ & $21 \mathrm{~s}$ & $7.7 \mathrm{~s}$ & 2048 & $57 \mathrm{~s}$ & $24 \mathrm{~s}$ & $1.2 \mathrm{~s}$ & 8 \\
9 & 6913 & 34561 & - & $56 \mathrm{~s}$ & $23 \mathrm{~s}$ & 4608 & - & $80 \mathrm{~s}$ & $2.9 \mathrm{~s}$ & 9 \\
10 & 15361 & 84481 & - & $160 \mathrm{~s}$ & $67 \mathrm{~s}$ & 10240 & - & - & $7.4 \mathrm{~s}$ & 10 \\
11 & 33793 & 202753 & - & $*$ & $214 \mathrm{~s}$ & 22528 & - & - & $19 \mathrm{~s}$ & 11 \\
12 & 73729 & 479233 & - & $*$ & $1254 \mathrm{~s}$ & 49152 & - & - & $53 \mathrm{~s}$ & 12 \\
\hline
\end{tabular}

TABLE 1.

The figures for AUTO and our trial implementation have been obtained using a SUN 3/60 with 16 $\mathrm{MB}$ of memory. The figures for Aldébaran, which are taken from [11], were obtained with a $50 \mathrm{MB}$ SUN 3/60. It is important to note that these figures refer only to the second phase of the algorithm where the strong bisimulation equivalence classes are computed. So the time it takes to carry out the first phase (the transitive closure) is not included. This means that, roughly speaking, the figures for Aldébaran must be multiplied by 2 . The figures for AUTO refer to the time needed for the complete algorithm. In separate columns the number of resulting equivalence classes of both experiments are given. They are the same for branching bisimulation and observation equivalence. In the table, "-." means that no outcome was obtained due to lack of memory and "*" means that no outcome is available.

Our implementation improves the performance of Aldébaran and AUTO considerably, especially when a lot of $\tau$ 's are around. For the space requirements this is directly reflected in the fact that, in the case where only the $a_{i}$-actions are visible, we can handle 12 cyclers on a $16 \mathrm{MB}$ machine, whereas Aldébaran, on a $50 \mathrm{MB}$ machine, can handle only 9 cyclers and AUTO, on a $16 \mathrm{MB}$ machine, only 8 . The figures about the time performance also show a considerable improvement (up to a factor 47). So it appears that in practical situations our algorithm is doing better than the usual algorithms for observation equivalence. However, we find it hard to draw firm conclusions from the results of our experiments since they are influenced by many uncontrollable factors. 


\section{REFERENCES}

[1] A.V. Aho, J.E. Hopcroft \& J.D. Ullman (1974): The design and analysis of computer algorithms, Addison-Wesley.

[2] T. Bolognesi \& S.A. Smolka (1987): Fundamental results for the verification of observational equivalence: a survey. In: Proceedings $7^{\text {th }}$ IFIP WG6.1 International Symposium on Protocol Specification, Testing, and Verification, Zürich. Switserland, May 1987 (H. Rudin \& C. West, eds.), North-Holland.

[3] M.C. Browne, E.M. Clarke \& O. Grumberg (1988): Characterizing finite Kripke structures in propositional temporal logic. Theoretical Computer Science 59(1,2), pp. 115-131.

[4] E.M. Clarke \& E.A. Emerson (1981): Synthesis of synchronization skeletons for branching time temporal logic. In: Proceedings of the Workshop on Logic of Programs, Springer-Verlag, pp. 5271.

[5] E.M. Clarke \& O. Grumberg (1987): Research on automatic verification of finite state concurrent systems. Ann. Rev. Comput. Sci. 2, pp. 269-290.

[6] E.M. Clarke, D.E. Long \& K.L. McMillan (1989): Compositional model checking. In: Proceedings $4^{\text {th }}$ Annual Symposium on Logic in Computer Science (LICS), Asilomar, California, IEEE Computer Society Press, Washington, pp. 353-362.

[7] D. Coppersmith \& S. Winograd (1987): Matrix multiplication via arithmetic progressions. In: Proceedings $19^{\text {th }}$ ACM Symposium on Theory of Computing, New York City, NY, pp. 1-6.

[8] R. De Nicola, U. Montanari \& F.W. VaAndrager (1990): Back and forth bisimulations, in preparation.

[9] R. De Nicola \& F.W. VaAndrager (1990): Three logics for branching bisimulation, to appear as: CWI Report CS-R90... Extended abstract to appear in: Proceedings LICS 90.

[10] E.A. EMERSON \& J.Y. HALPERn (1986): 'Sometimes' and 'Not Never' revisited: on branching time versus linear time temporal logic. JACM 33(1), pp. 151-178.

[11] J. Fernandez (1989): An implementation of an efficient algorithm for bisimulation equivalence.

[12] R.J. VAN GlabBeEK \& W.P. WeiJland (1989): Branching time and abstraction in bisimulation semantics (extended abstract). In: Information Processing 89 (G.X. Ritter, ed.), Elsevier Science Publishers B.V. (North Holland), pp. 613-618.

[13] R.J. van Glabbeek \& W.P. Weilland (1989): Refinement in branching time semantics. Report CS-R8922, Centrum voor Wiskunde en Informatica, Amsterdam, also appeared in: Proceedings AMAST Conference, May 1989, Iowa, USA, pp. 197-201.

[14] P.C. Kanellakis \& S.A. Smolka (1983): CCS expressions, finite state processes, and three problems of equivalence. In: $2^{\text {nd }} \mathrm{ACM}$ Symposium on Principles of Distributed Computing (PODC), Montreal, Quebec, Canada, August 1983, to appear in: Information \& Computation.

[15] L. LAMPORT (1983): What good is temporal logic?. In: Information Processing 83 (R.E. Mason, ed.), Elsevier Science Publishers B.V. (North Holland), pp. 657-668.

[16] R. Milner (1980): A Calculus of Communicating Systems, LNCS 92, Springer-Verlag.

[17] R. Milner (1989): Communication and concurrency, Prentice-Hall International.

[18] R. PAIGE \& R. TARJAN (1987): Three partition refinement algorithms. SIAM Journal on Computing 16(6), pp. 973-989.

[19] R. de Simone \& D. Vergamini (1989): Aboard AUTO. Technical Report 111, INRIA, Centre Sophia-Antipolis, Valbonne Cedex. 
